\title{
La diplomacia como estrategia de sometimiento indígena en la frontera este del Tucumán (1700-1750)
}

\begin{abstract}
ALBERTO J. GUILLON ABAO
Al igual que en otras zonas del continente americano, los españoles asentados en la Gobernación del Tucumán se vieron obligados a mantener duros enfrentamientos con las poblaciones indígenas para conseguir un espacio donde poder desarrollar su estructuras políticas, sociales y económicas. Pero este proceso, ante las precarias condiciones de los asentamientos iniciales españoles en.el área, fue de larga duración, siendo uno de los elementos fundamentales para su permanencia -junto a los presidios y las reducciones- "las paces y los tratados" establecidos con los caudillos indígenas.
\end{abstract}

\section{LAS PETICIONES DE PAZ}

La política de tratados fue una constante en los tres siglos de dominación española, aunque se incentivó en el último. El primer tratado de paz que conocemos en el S. XVIII fue el que estableció el lugarteniente de la ciudad de Santiago del Estero, Alonso de Alfaro, con los lules en 1703, aunque unos años antes ya hubo algún intento por parte del gobernador Tomás Felix de Argandoña de entablar tratados de amistad con algunos pueblos chaqueños (1). Posteriormente, el gobernador Urízar y Arespacochaga comunicó al Rey el sometimiento de numerosos pueblos chaqueños, entre los que estaban.los tobas, malbalaes, ojotaes, isistines, toquistines, etc..., con quienes habían firmado diversas paces (2). El gobernador Santiso y Moscoso informó sobre la petición de paz por parte de los tobas, así como respecto a los acuerdos que obtuvo con ellos. Y los gobernadores Martí-

(1) Petición presentada por los capitanes y reformados ante el maestre de campo Ignacio. Ibáñez, siendo teniente del fuerte de Yuquiliguala. 6-noviembre-1690. AGPSE. A.G. Contaduría 1730/803. El gobernador Gaspar de Barahona sobre las paces establecidas con los indios lules cuando estaban reparando el cauce del rio Salado. Salta 6-diciembre-1703. AGPSE. AG. Legajo 1, expd 3.

(2) El gobernador de Tucumán da cuenta de las dos campañas que ejecutó contra las naciones bárbaras del chaco, los indios que se rindieron y los tres fuertes que puso de avanzada para mayor seguridad. Salta 24-Julio-1712. AGI. Charcas 284.

DOI: http://dx.doi.org/10.25267/Trocadero.1995.16.17 
nez de Tineo y Espinosa y Dávalos dieron noticias sobre las naciones chaqueñas que habían llegado a solicitar las paces, firmando con ellos diversos tratados.

Esto no supuso el abandono de políticas paralelas de dominación como expediciones armadas o establecimiento de presidios y reducciones, máxime cuando la mayoría de las peticiones de paz por parte de los indigenas chaqueños se produjeron cuando los españoles estaban batiendo el territorio fronterizo; así, en numerosas ocasiones observamos como los chaqueños a la hora de pedir sus paces hablaban del desasosiego contínuo que sufrían a causa de las expediciones españolas, careciendo de seguridad y sin poder alimentarse durante días por haberse ocultado en los montes como medida de precaución; también se discurría sobre el temor que tenían a algunos "pueblos amigos", tales como las chiriguanos, quienes sembraban auténtico pavor entre las filas de los tobas del norte (3).

Sin embargo, en otras ocasiones fueron los propios indígenas los que solicitaron los tratados, que generalmente ya tenían apalabrados anteriormente con los españoles, como ocurrió con los vilelas en 1728, cuando cinco chaqueños pidieron que se estableciesen las paces prometidas el año anterior (4). Esta actitud por parte de los indigenas respondió generalmente a las presiones que ejercieron los expedicionarios españoles, obligando a los pueblos del Chaco a desplazarse al interior y disputar con sus "enemigos" ancestrales los territorios de caza y pesca, procurando que los menos fuertes buscasen la supuesta seguridad que les ofrecía pactar con los españoles. En otras oportunidades las peticiones de paz fueron consecuencia de la captura de algún individuo de una parcialidad indígena con cierto prestigio entre su pueblo (un jefe, el hijo de un curaca, uno de los capitanes,...), tal como ocurrió en Santiago del Estero en 1746, cuando los hombres al mando de Francisco de la Barreda asaltaron unos "toldos", capturando a la mujer de un español cautivo, que vivía con los abipones desde pequeño, y éste para recuperarla optó por ayudar en el establecimiento de un tratado de paz con el cacique Alaiquín y su pueblo, llevando implícita la paz el canje de cautivos (5).

\section{EL PROTOCOLO}

Las paces se establecieron siguiendo un modelo similar a otras zonas americanas (6). En primer lugar los españoles hacían una junta de guerra donde decidian si admitían o no las paces de los indios, distinguiendo entre los que habían

(3) Gould, Eduardo y Lobos, Héctor. Contribución al estudio de la frontera de la gobernación del Tucumán con el Chaco durante el gobierno de Angel de Peredo (1670-1674). Inédito. Córdoba, 1989, pág. 12.

(4) Levene, Ricardo y Gargaro, Alfredo. Actas capitulares de Santiago del Estero. T.I Buenos Aires, 1941, pág. 101-102.

(5) Achaval, Nestor José. Historia de Santiago del Estero siglos XVI-XIX. Santiago del Estero, 1988, pág. 179. El obispo de Tucumán, Pedro Miguel de Argandoña, en respuesta a la carta de Francisco Barreda sobre la reducción de los abipones. Córdoba-17-mayo-1749. AGPSE. Temporalidades 1724-750.

(6) Mendez Beltran, Luz María. La organización de los parlamentos de indios en el siglo XVIII. En "Relaciones fronterizas en la Araucania". Santiago de Chile. 1982. 
"insultado" (aquellos que habían asaltado estancias, haciendas, pueblos, etc...) y los que no. Fueron estos últimos, según Lozano, a los que se les podía ofrecer la paz, aunque la realidad fue que sí los indígenas la solicitaban, fuesen unos u otros, se le aceptaba por regla general (7). En las ocasiones en que fueron los indios quienes se presentaron en las ciudades proponiendo la paz, se celebraba un cabildo abierto para determinar sobre el asunto (8). Tanto en la junta de guerra como en los cabildos, se cotejaban diversas opiniones sobre la posibilidad de dichas paces, sitios donde ubicar a los indígenas, fondos disponibles, si eran aptos para la reducción, etc...; pero era el gobernador quien tenía la última palabra.

En las paces que se firmaron con los indígenas que salieron voluntariamente del Chaco, hemos de suponer que existieron acuerdos anteriores entre ellos, sopesando los pro y los contra que les ofrecía la amistad con los españoles, lo que hasta cierto punto explicaría que varios caciques o capitanes de distintas naciones pidieran reducción bajo un mismo líder, con unos objetivos realmente definidos, sobre todo en la segunda mitad del siglo XVIII, como en las paces celebradas con el cacique Paikín (9).

En la mayoría de los casos, los chaqueños entregaban a familiares directos de los caciques como rehenes en garantía de amistad y de cumplimiento de su palabra; además, traían con ellos cautivos para darlós en prueba de buena voluntad, que al mismo tiempo les servían como intérpiretes. Los españoles también utilizaban lenguaraces, que por lo general eran cautivos, aunque también hubo blancos y mestizos que se profesionalizaron en este oficio, tras haber aprendido generalmente la lengua al ser capturados de péqueños, vivir en zonas fronterizas o estar prófugos de la ley refugiados en alguna toldería. En otrás oportunidades estos intérpretes eran los misioneros que habían aprendido la lengua de los indigenas reducidos.

La segunda fase del tratado de paz era la junta de deliberación o la junta con los chaqueños. En el casó de la petición de paz por parte de los indígenas, una vez recibidos en el cabildo, se les interrogaba y preguntaba sobre las causas y motivos por los que deseaban la paz. En el caso que el tratado se firmase como consecuencia de una entrada directa de españoles en el territorio chaqueño, se les "..invitaba a vivir en una vida política y ordenada..", y a que conocieran el "..verdadero y único Dios..", exhortaciones y formulas que fueron, para algunos autores, un auténtico requerimiento (10), imponiéndoles una paz que en la mayoría de los casos los indígenas aceptaron como estrategia de supervivencia, al verse acosados por la presencia inmediata de las tropas expedicionarias.

Posteriormente se establecian los capítulos de paz, separándolos en dos grandes bloques: por un lado las obligaciones (también llamadas "capitulaciones"), que los indígenas contraían con los españoles; y por otro las contrapartidas que iban a recibir los chaqueños, todo ello acompañado de duras sanciones para

(7) Lozano, Pedro. Descripción corográfica del gran Chaco Gualamba. San Miguel de Tucumán, 1944, pág. 318.

(8) Levene, Ricardo y Gargaró, Alfredo. Actas capitulares de.... Opus cit, págs. 101-102.

(9) Paces celebradas entre el gobernador Gerónimo de Matorras y las naciones mocovies y tobas en los paises del Gran Chaco 29-julio-1774. AHT. Administrativa. T. VII, fols. 304-307.

(10) Vitar, Beatriz. Las relaciones entre los indígenas y la sociedad colonial en un espacio conflictivo: La frontera tucumana chaqueña en el siglo XVIII. En "Congreso de Etnohistoria". Inédito. Buenos Aires 1989. 
los indígenas que infringieran los acuerdos. A continuación se pedía la aprobación del representante de la parcialidad, que tras aceptar las condiciones "..se fundía en abrazos, saludos.." y un sin fin de manifestaciones externas de paz. Todo esto se realizaba con gran teatralidad, existiendo órdenes específicas del jefe de los expedicionarios de manifestar buen trato y compostura, entregando regalos, alimentos y presentes, con el fin de impresionar a "los salvajes".

Algunos autores escriben que el simple hecho del establecimiento de las paces implica un reconocimiento de la libertad de los indígenas, e incluso Lizondo Borda llega a afirmar que en el tratado de los tobas de 1742 , con el gobernador Santiso y Moscoso "... fue acaso la primera vez que los orgullosos españoles trataban con respeto y como a sus iguales no solamente a una nación indígena sino a personas de indios" (11). Pero a pesar de dicha afirmación, las paces reflejaban la intención de introducir los valores más intrínsecos de los españoles (único Dios, Rey, justicia española...), en el mundo cultural indígena, con el fin de aculturarlos e insertarlos en el orden colonial.

\section{LAS PACES CELEBRADAS: OBLIGACIONES Y PRIVILEGIOS}

Para analizar el contenido de los distintos pactos firmados entre españoles e indios hemos tomado varios ejemplos: el tratado establecido en 1703 con la nación de los lules; el de 1710, con la nación de los malbalaes; el de 1742, con los tobas; y el de 1750 , de nuevo con los malbalaes. Se puede observar que el tratado de 1710 con los malbalaes contiene condiciones mucho más severas, en su conjunto, que el antecedente de 1703 con los lules, pareciendo más un acto de perdón y benevolencia por parte de los españoles que un auténtico tratado de paz. Esto se puede explicar porque el acuerdo de 1710 fue firmado en pleno Chaco, acosados los malbalaes por las huestes españolas, y porque esta nación era considerada como muy peligrosa, ya que en múltiples ocasiones había atacado y destruido estancias y haciendas en toda la provincia. Sin embargo, los lules también atacaron a los españoles, asaltando el Camino Real -que unía Buenos Aires con el Alto Perú- coaligados con los mocovies (12), lo qué nos llevó a preguntarnos sobre las causas por las que este pueblo obtuvo mejores condiciones en las paces. Probablemente habria que buscarlas en la experiencia que los lules ya tenían, puesto que habían sido encomendados con anterioridad y se vieron sometidos a un régimen de sobrexplotación que acabó con muchos de ellos; de ahí que, sí querían pactar con ellos, sus condiciones debían ser aceptadas tal y como la formularon: no ser encomendados, no pagar tributo, permitir o no las "entradas" de doctrineros, etc.. (13).

(11) Lizondo Borda, Manuel. El Tucumán de los siglos XVII y XVIII. En "Historia de la Nación Argentina". Vol. IrI. Buenos Aires, 1939 fol. 293. Zapater, Horacio. Parlamentos de paz en la guerra del Arauco. En "Arucania. Temas de historia fronteriza". Santiago de Chile, 1985, págs. 47-82.

(12) Petición presentada por los capitanes y reformados ante el maestre de campo Ignacio Ibáñez, siendo teniente del fuerte de Yuquiliguala. 6-noviembre-1690. AGPSE. A.G. Contaduría $1730 / 803$

(13) El gobemador Gaspar de Barahona informa sobre las paces establecidas con los indios lules cuando estaban reparando el cauce del rio Salado. Salta 6-diciembre-1703. AGPSE. AG. Legajo 1, expd. 3. 
Las dos paces posteriores fueron tratados firmados a mediados de siglo, donde existía ya por parte de los españoles un mayor conocimiento de ambas naciones; y sabían de su inconstancia a la hora de mantener los pactos establecidos. Se perseguía controlarlos y atraerlos a "la vida cristiana y política", pero manteniéndolos a distancia y bien supervisados.

Si estudiamos las obligaciones impuestas a los indígenas en los cuatro tratados analizados, en todos' los casos se prefijaba que debían ser "fieles y leales vasallos", y obedecer a las autoridades de la provincia, pero en la paz con los lules de 1703 observamos que esta premisa estaba inserta en el marco de los derechos específicos, ya que el simple hecho de ser vasallo implicaba una protección por parte de la legislación española. La cuestión defensiva abarca varios puntos de estas obligaciones: se trataba de una paz perpetua y de un pacto de alianza en caso de ataque, al mismo tiempo que debían reconocer a los indios amigos de los españoles como propios, y brindarles auxilios en la medida de sus posiblitidades. Estas obligaciones también implicaban informar sobre las operaciones de los mutuos enemigos, actuando como espías, rescatando y devolviendo el mayor número posible de cristianos cautivos que tuviesen otras naciones, y no comunicarse con los posibles enemigos del interior del Chaco sin la supervisión de las autoridades.

En el apartado dedicado a la "fé", se establecía que debían admitir la religión y sus predicadores, comprometiéndose a aprender la doctrina y política cristiana cumpliendo las órdenes que en tal sentido se les impartieran. Este último punto tiene variaciones en los diferentes tratados: en el firmado con los lules se estipulaba la voluntariedad de recibir los misioneros, y en el caso de los tobas se consideró que todavía no estaban convencidos ni persuadidos para formar reducción con ellos, por lo que debían mantenerse en los límites fronterizos, aunque sî debían permitir a los misioneros entrar a convertirlos.

Otro de los puntos básicos fue la ubicación de los indígenas en un territorio concreto, para permitir un mejor control de los españoles sobre los mismos, disminuyendo así una movilidad que hacía tan difícil cualquier tipo de proceso de aculturación. A los tobas se les obligó tan sólo a ubicarse en una zona cercana a los presidios, ya que la experiencia había demostrado a los españoles el riesgo que representaba tener cerca de las ciudades a pueblos tan belicosos. En los dos últimos tratados, de 1742 y 1750 , se especificó que no podían introducirse en las fronteras más de dos personas, que debían explicar previamente los motivos para hacerlo, otorgándoles para ello un "pasaporte de amigos de paz y comercio". Se pretendía, de esta manera, evitar las posibles reuniones o malones detrás de la línea defensiva de los fuertes para atacar las ciudades o jurisdicciones cercanas. En el caso concreto del tratado con los tobas de 1742, se les impusieron algunas condiciones realmente humillantes: podían ser aprehendidos si fuesen encontrados sin cumplir algunas de las normas; los soldados españoles debían ir a sus tierras e inspeccionar, sin que les pusiesen ningún tipo de oposición; y si por algún error se ejecutase castigo a dicha nación, o a parte de ella, no se ofenderían ni se sentirían agraviados.

A cambio de ello, los indígenas recibirían la "constante" palabra de amistad de los españoles, quienes cumplirían con todos los acuerdos estipulados con estas naciones; mantendrían a los caciques y mandones con su dignidad y como interlocutores, defendiendo sus pueblos en caso de ser atacados por los enemi- 
gos comunes; no podían ser agraviados ni insultados por los españoles o por otras naciones indígenas y, si así fuese, serían castigados los infractores por la justicia española. Se comprometieron también a ayudarles en todas sus necesidades, dándoles personas que les intruyesen en las labores de los campos, construcción de casas, arreo de ganado, etc... En el caso particular de los malbalaes de 1712, se les perdonaban los delitos que, según los españoles, habían cometido desde mucho tiempo atrás y no se hacía referencia alguna a la encomienda; a diferencia de las claúsulas que, como ya hemos dicho antes, los lules consiguieron (14).

\section{¿QUÉ SE BUSCABA EN LAS PACES?}

Como ya hemos expuesto en trabajos anteriores (15), las políticas fronterizas que los españoles estaban realizando no obtuvieron los frutos deseados, los chaqueños no sometidos continuaron incursionando en la jurisdicción de la Gobernación. Además, los premios que los expedicionarios obtenían fueron más escasos a medida que pasaban los años, siendo las encomiendas cada vez más difíciles de obtener. El reparto de piezas cautivadas era cada vez menor, lo que no llegaba a justificar los gastos que reportaban las expediciones y ni siquiera constituían ya un aliciente lo suficientemente importante para que los hombres se interesasen por salir a las campañas que se realizaban. A ello hemos de sumarle los distintos impuestos para mantener hombres en la frontera, que nunca eran suficientes, teniendo los pobladores que seguir aportando la ayuda financiera y milicianos para la defensa. Por todo ello, y por los buenos resultados que habían obtenido los españoles en otros territorios americanos, creyeron que estableciendo buenas relaciones con varios pueblos chaqueños, estos atraerían a sus "hermanos" del interior, con lo que finalizaría una guerra que se eternizaba, con enormes gastos para los particulares, no sólo en el aporte expedicionario (víveres, caballos, armas...), sino también en pérdidas económicas por el abandono de sus tierras para ir al Chaco (16).

Se pretendió la colaboración de los indios en el ámbito militar, y se quiso establecer un cordón de pueblos "aliados" en la frontera que sirviera de protección contra las naciones del interior (17).

La ubicación en un sitio concreto cercano a los fuertes de estas poblaciones permitió a los españoles un cierto control de sus movimientos, intentando evitar las posibles confederaciones con los indígenas del interior, así como un mayor conocimiento de los usos y costumbres de estas naciones. Se privilegió a los líderes de estos grupos, dejándoles continuar con la jefatura y afirmándolos en su poder. Con ello se pretendió conseguir un interlocutor capaz de representar a su

(14) Ibidem. Tratado de paz entre el gobernador Juan de Santiso y Moscoso y los indios tobas. Salta 12-junio-1742. AGPSE. AG. Legajo 2, expd. 114. Lozano, Pedro: Descripción corográfica... Opus cit, pág. 343. Capitulaciones que deben observar los indios malbalaes, nuestros amigos reducidos. Campo del Rio Negro 27-agosto-1750. AGI. Buenos Aires 303.

(15) Gullón Abao, Alberto J.: La frontera del Chaco en la Gobernación del Tucumán (1750-1810). Cádiz, 1993.

(16) Gullón Abao, Alberto J.: La Gobernación del Tucumán en la primera mitad del S. XVIII. Coacciones y reacciones ante una guerra de fronteras. En "Trocadero" $\mathrm{n}^{\circ}$. 4, 1992.

(17) Levene, Ricardo y Gargaro, Alfredo. Actas Capitulares.... Opus cit, págs. 175-177. 
comunidad a la hora de peticiones concretas, pero, especialmente para que hiciese cumplir en el seno de las parcialidades los designios de la administración colonial.

También se contempló, sobre todo a mediados del siglo XVII, la posibilidad de explotación de estas naciones reducidas como mano de obra barata y estacional, lo que impulsó aún más la política de paces y tratados. Es el caso, por ejemplo, de los grupos mataguayos que trabajaron "voluntariamente" como peones conchabados en las siegas de trigo, obras públicas de las ciudades, corte de la caña en las haciendas, 'etc. Por otra parte, otros factores incidieron sobre la firma de estos acuerdos: el establecimiento de relaciones comerciales más o menos estables aportó un mayor conocimiento de la cultura de estas naciones, por lo que se comprendió que era difícil, con los medios disponibles, vencerles en toda la línea; además, fueron un posible mercado para algunos productos, al mismo tiempo que se obtuvieron del Chaco "productos de la tierra" (miel, cueros, etc..); sin olvidar tampoco la oposición manifiesta de algunas ciudades a las salidas al Chaco por su cada vez menor eficacia (18).

Uno de los grupos que mas presión ejerció para que se efectuase esta política de paces y tratados fue la Iglesia, y en especial la Compañía de Jesús, que desde finales del siglo XVII intentó reducir a los indígenas chaqueños, a quienes, con su política paternalista y de separación del blanco, los protegía de los españoles que pretendían su explotación como mano de obra. Fueron los jesuitas quienes más apoyaron la política de pacificación en el Chaco, asegurando que éstos defenderían la frontera con mucho menos gasto que los partidarios y los cuerpos expedicionarios, siendo su único costo el alimento que habría que proporcionarles en un primer momento a los reducidos; después, aprenderían los principios de la agricultura y ganadería, logrando autoabastecerse. Además se atraérían a los indigenas no reducidos si estos veían cierto éxito en las reducciones, y se aseguraría el principio básico y principal de la Corona, que según ellos, era la conversión de estos "infieles" a la fe cristiana (19). El resultado fue un fuerte proceso de aculturación y, especialmente tras la expulsión de los jesuitas, la desarticulación del universo político, social, económico y religioso del indígena, que posibilitó a los españoles la obtención de una mano de obra acorde con las necesidades del mercado laboral, así como nuevas tierras; proceso que culminó a fines del siglo XVIII y principios del XIX con el trabajo de los indígenas en las haciendas azucareras, y la explotación y venta de las tierras antaño de las reducciones.

También los indígenas obtuvieron ciertos beneficios de los tratados. En el campo bélico, la alianza con los españoles les permitió participar en las distintas expediciones contra sus enemigos ancestrales, apoyados por la superioridad que representaban los caballos; "bocas de fuego" y aplastante número de hombres, obteniendo parte del botín, caballos, ovejas, armas, etc, y vendiendo las piezas

(18) Certificación del teniente general, justicia mayor y capitán a guerra, José Arias Rengel, y varios oficiales militares, sobre la tranquilidad y paz de la provincia lograda tras la campaña pasada. Salta 6-diciembre-1760. AGI. Buenos Aires 49.

(19) El P. Juan Reus, doctrinero de la Compañía de Jesús; informando al Gobernador sobre las necesidades de ganado para la reducción de Jesús, María y José. Presidio de San Fernando 1-septiembre-1745. AGN. S.IX 5-6-6. 
que capturaban (20). Al mismo tiempo, los españoles se comprometían a su defensa en caso de ser atacados por otros pueblos rivales. Los chaqueños reducidos también usaron las paces para ocultar sus correrías; así, los de Santa Fe, amparándose en ellas, asaltaron la jurisdicción de Córdoba, vendiendo en la ciudad lo obtenido, con cierto beneplácito de un sector de la población, que vió un buen negocio en la compra de los objetos y productos robados. En otras ocasiones fueron los abipones de la reducción de Concepción, en la jurisdicción de Santiago del Estero, quienes coaligados con los del interior, asaltaron las estancias vecinas, buscando luego refugio en dicha reducción sin recibir castigo alguno por temor a un levantamiento general (21).

Otro de los factores que influyó en la decisión de los indígenas para aceptar las paces fue la seguridad en la obtención del alimento. Estas tribus nómadas que subsistían de la caza, pesca y recolección en su mayoría, tenían una gran dependencia del medio a la hora de obtener el sustento necesario para su supervivencia, siendo relativamente frecuentes las hambrunas por cuestiones climatológicas, ya fuesen sequías o inundaciones, y la posibilidad de obtener alimentos (ganado vacuno, trigo, maíz, etc...) fue la piedra angular en la atracción de estas parcialidades (22). También consiguieron, una vez firmadas las paces, una serie de materiales difíciles de obtener (agujas, hachas, cuñas de hierro, cuchillos...). Junto a estos, un $\sin$ fin de menudencias, vidrios, brazaletes de metal, pendientes..., que tenían connotaciones rituales o de representación social; y por supuesto telas, casacas, pantalones, paños,..., de llamativos colores; todo ello sin correr el riesgo de franquear la línea defensiva española, y sin necesidad de exponer la vida en los asaltos a las carretas que pasaban por el camimo real, o a las estancias cercanas, cada vez mejor defendidas por los colonos (23).

Se les otorgó terrenos en sitios relativamente cómodos, y con abundante caza y pesca para la subsistencia, mientras crecían las sementeras. Obtuvieron una casa, o tancho, con tierra propia para hacer huerta y un corral, además de las tierras comunales que debían eligir de mutuo acuerdo españoles e indígenas. La cercanía del Chaco les permitía recoger los productos tradicionales, como la cera, miel, algarroba, etc.., así como pescar y cazar, complementando su dieta y vendiendo la mayor parte del producto de la recolección, aunque a precios mínimos. Estas fueron las llamadas reducciones, que aislaron durante bastante tiempo la república de españoles y la república de los indios, por la labor de los jesuitas y posteriormente de los franciscanos (24).

(20) Carta del maestre de campo Antonio de la Tijera al gobennador Esteban de Urízar y Arespacochaga, sobre la situación de la frontera y la ayuda que pueden presentar los tobas. Fuerte del Rosario 29-febrero-1712. AHPJ. Cajón XL, legajo 3, pág. 18 vta.

(21) Informe del gobernador Joaquín de Espinosa al virrey José Antonio Manso, sobre la muerte de treinta personas en el camino de los Porongos. Salta 22-septiembre-1758. AGN. S. IX 5-6-6.

(22) Informe de Francisco de la Barreda sobre la reducción de Concepción de Abipones. Santiago del Estero 5-enero-1750. AHPT. Administrativa. T.III, fol. 3.

(23) Wayne Powell, Philip. El capitán mestizo: Miguel Caldera y la frontera norteña. La pacificación de los chichimecas (1548-1597). México, 1980. pág. 182.

(24) Carta del gobernador Victorino Martinez del Tineo, al comandante de partidarios, sobre la creación de una reducción para los indios malbalaes. Trampa del tigre 21-julio-1750. AGI. Buenos Aires 303 . 
A ello hemos de sumar que se produjo un mayor conocimiento por parte de los indígenas: de sus "aliados" españoles, lo que les permitió obtener información sobre sus armas de fuego, las estrategias que usaban, sus fortificaciones, y. los caminos que más facilmente permitían el acceso a las estancias, cuales de ellas eran más ricas, y cuales entrañaban menos riesgos a la hora de un posible asalto. La información obtenida fue utilizada con frecuencia tras la ruptura de estos pactos, con resultados bastante satisfactorios para los chaqueños (25).

\section{DE LA TEORIA A LA PRACTICA}

Pero las paces y los tratados no obtuvieron los resultados esperados en un primer momento, ya que la ruptura de estos estaban intrinsecamente unidos, generalmente, a las condiciones en que se estipularon dichos acuerdos y a las obligaciones que contenían. Entre las causas más corrientes de la ruptura de los pactos se destaca el que los indígenas, al verse amenazados en su propio territorio por fuerzas muy superiores en número y con tecnología superior, se vieron obligados a someterse y a pedir dichos tratados, pero más como una estrategia de supervivencia que como un acto voluntario, esperando la primera ocasión para sublevarse. Por ello no resulta extraño que los malbalaes reducidos por el gobernador Urízar atacasen el fuerte que les pusieron cercano a la reducción, poco después de firmar dichas paces (26); años después, algo similar ocurría al gobernador Victorino Martínez de Tineo con el mismo pueblo (27).

La falta de un jefe que aglutinase estas naciones en su totalidad, hizo que los españoles se aliasen a caciques o capitanes, que sólo eran representativos de una parcialidad, y que no dudaron en romper dichos pactos, apoyando a los suyos u otros pueblos amigos de antaño, asaltando las haciendas con sus confederados (28).

Se pretendió, con gran desconocimiento e ingenuidad por parte de las autoridades coloniales, que naciones enemigas inmemoriales se volviesen aliadas para luchar contra otros pueblos, y al mismo tiempo que viviesen en paz, lo que causó graves conflictos, teniendo que separarlos en las reducciones por naciones, pues las luchas y tensiones entre ellos fueron frecuentes.

Tambien fue común el incumplimiento por los españoles de las condiciones firmadas con los indígenas, principalmente en las claúsulas referidas a su alimentación; el ganado, trigo y maíz que los españoles les daban, no cubrieron las mínimas necesidades de los recién reducidos, y más aún cuando se les incorpora-

(25) El gobernador Victorino Martínez de Tineo, informa sobre la huida que ha efectuado los indios malbalaes recién reducidos. Salta 12-junio-1751. AGI. Buenos Aires 303.

(26) El gobernador Esteban de Urizar y Arespacochaga, informa de las dos campañas que ejecutó contra las naciones bárbaras, los indios que se rindieron y los tres fuertes que puso de avanzada para mayor seguridad. Salta 24-julio-1712. AGI. Charcas 284, fol. 205.

(27) Carta del gobernador Victorino Martinez de Tineo al comandante de partidarios, sobre la creación de una reducción para los indios malbalaes. Trampa del tigre 21-julio-1750. AGI. Buenos Aires 303.

(28) Petición presentada por los capitanes y reformados ante el maestre de campo Ignacio Ibáñez; siendo teniente del fuerte de Yuquiliguala. 6-noviembre-1690. AGPSE. A.G. Contaduría $1730 / 803$. 
ba un creciente número de indios recién salidos del Chaco. A lo que debe agregarse el terrible choque cultural que suponía para el universo indígena el proceso de aculturación a que se vieron sometidos en el ámbito político, económico, social y religioso, lo que provocó reacciones violentas contra los españoles, reflejadas en el abandono de las reducciones, asaltos a los fuertes, muertes de los misioneros, desidia ante las labores encomendadas, etc... (29).

No todos los españoles estuvieron de acuerdo con esta política. Existió un sector en la sociedad que no creía en los pactos que se pudiesen establecer con los indígenas, y que veían como única solución al problema la guerra de exterminio y la captura del mayor número de indígenas, repartiéndolos entre los vecinos de las ciudades y hacendados, o desnaturalizándolos de sus tierras. Fueron los que apoyaron la continuidad de la guerra y la venganza como única respuesta ante las incursiones. Estas opiniones se escucharon cuando se produjeron las rupturas de las paces, provocando fisuras en la política de pacificación por medio de pactos. A pesar de ello, prevalecieron los intereses de los partidarios de esa política: el clero regular; las autoridades encargadas de la Real Hacienda, ya que la guerra resultaba mucho más onerosa para el fisco; los comerciantes, que veían la posibilidad de establecer un camino libre, sin los cuantiosos gastos que les reportaban las escoltas y los impuestos para la defensa; y los hacendados, que observaban todos los años cómo la mano de obra tenía que abandonar el trabajo para salir a las contiendas. A ello deben unirse los intereses de la Corona, que esperaba aplacar la violencia de la frontera, extender la "policia" de sus habitantes y el buen gobierno, por lo que siguió apostando por la política de paces, apoyada por los fuertes y reducciones, que fueron afianzándose en la segunda mitad del siglo XVIII.

(29) Carta del gobernador Martinez de tineo, informando de los progresos logrados en su entrada general con los tercios de las ciudades. Salta 16-octubre-1750. AGI. Buenos Aires 303. 